\title{
A Note on the Fractal Behavior of Hydraulic Conductivity and Effective Porosity for Experimental Values in a Confined Aquifer
}

\author{
Samuele De Bartolo, Carmine Fallico, and Massimo Veltri \\ Department of Civil Engineering, University of Calabria, 87036 Rende, Italy \\ Correspondence should be addressed to Samuele De Bartolo; samuele.debartolo@unical.it
}

Received 16 October 2013; Accepted 21 November 2013

Academic Editors: P. López-Arce, W. O. Wong, and Q. W. Yang

Copyright ( 2013 Samuele De Bartolo et al. This is an open access article distributed under the Creative Commons Attribution License, which permits unrestricted use, distribution, and reproduction in any medium, provided the original work is properly cited.

\begin{abstract}
Hydraulic conductivity and effective porosity values for the confined sandy loam aquifer of the Montalto Uffugo (Italy) test field were obtained by laboratory and field measurements; the first ones were carried out on undisturbed soil samples and the others by slug and aquifer tests. A direct simple-scaling analysis was performed for the whole range of measurement and a comparison among the different types of fractal models describing the scale behavior was made. Some indications about the largest pore size to utilize in the fractal models were given. The results obtained for a sandy loam soil show that it is possible to obtain global indications on the behavior of the hydraulic conductivity versus the porosity utilizing a simple scaling relation and a fractal model in coupled manner.
\end{abstract}

\section{Introduction}

The scale concept is strongly related to parameters characterizing flow and transport in porous media, mainly to hydraulic conductivity, for which the scale behaviour was widely verified in the literature [1-11], and to the role of this related to the effective porosity, which strongly influences flow in porous media [12-22]. The causes of the scaling behaviour are generally attributed to the medium heterogeneity $[7,23,24]$. Specifically, it was noted that different scales (laboratory scale, field scale, regional scale, and so on) could be considered according to the specific problem investigated and to the type or the particular method of measurement considered. Furthermore, at a different scale, the manner in which the heterogeneity influences the scale behavior is generally different, mainly the shape and size of pores, from small scales and their continuity from larger ones $[12,16$, $18,19,22,25-27]$. In this framework the effective porosity, with other parameters such as the tortuosity and the pore network connectivity, plays a fundamental role with regard to the water flow in the porous medium. In any case the scale behavior of the effective porosity is a topic that should again be well characterized in the proper measurement scale and in the other contexts of the scales involving the measurement of the same hydraulic conductivity [28-37]. Therefore, below we will refer exclusively to that parameter $(\varphi)$.

To describe the scale behavior the majority of the studies on this topic consider power type laws. However, even this aspect requires further investigation and clarification, especially considering that often the data set of the parameter in question is composed of subsets achieved by different measuring methods, which generally involve aquifer volumes of different sizes and hence different scales. The relative reference scale varies also for increasing aquifer volumes, as a result of the different ways in which the heterogeneity influences the phenomenon. This occurs in all real aquifers, many of which are often considered homogeneous in the absence of stratifications or obvious differences related to geological hazards. The assumption for the porous medium of the scale homogeneity is well suited to the hypothesis of self-similarity, with respect to which the fractal models can be taken into account $[22,36]$.

With reference mainly to the hydraulic conductivity, many authors $[9,38,39]$ took into account the scaleinvariant behaviour of a well-defined measurement range and addressed the scaling study and the description of the hydraulic conductivity and the effective porosity. Specifically, the relationship between these two quantities in terms of 
measurement, in most cases represented by laws of power type and fractal models, has often concerned well-defined ranges of scale in the context of the measures taken. However, some problems still remain open regarding the definition of scaling cut-off limits, if these exist, with respect to which the measurement scale adopted may well be considered representative. This aspect can be examined in terms of simple-scaling and multiscaling behavior but does not exclude the possibility of interpretation of these scaling behaviors by other laws, also different from those of the power type [40].

The simple and multiscaling behavior of hydraulic conductivity in the last two decades was put in relation both in terms of porosity and in terms of measurement scale. The description of this behavior in terms of power law, among many works in the literature, can be traced to the early experiences of Jacquin and Adler [12] and Muller and McCauley [41] on the generalization of the Kozeny-Carman equation in terms of fractal geometry (geometry of porous geological structures) if the process is considered in the pore scale, until recent studies provided by $\mathrm{Xu}$ and $\mathrm{Yu}$ [36]. While for the scale of measurements (including aquifer tests) reference works can be traced to those of Neuman [4], Rovey II and Cherkauer [5], Guimerà et al. [6], Schulze-Makuch, and Cherkauer [8]. As part of the relationship between hydraulic conductivity $(k)$ and porosity $(\varphi)$ the basic equations had also a review in terms of pore-space fractal geometry assumption [22], achieving good results for nongranular systems (fiber mats and vesicular rocks). Developments related to further scales (laboratory and field) can be considered, such as those of Giménez et al. [16], Pape et al. [18], Giménez et al. [19], and Regalado and Muñoz-Carpena [21]. Some considerations can be made for the effective porosity, which is a structural parameter of fundamental importance for the description of the water flow in porous media. However, the scaling behaviour of the effective porosity still requires further investigation and details in order to consider the topic sufficiently characterized, as pointed out by numerous studies examining also the relationship between this parameter and hydraulic conductivity and the context of the scales involving the measurement of these two parameters [28-37].

The use of fractal models can provide important guidelines on the determination of the physical scaling relative to the measurement scale. This specifically concerns the functional link between porosity and scale and between hydraulic conductivity versus porosity, which is equivalent to the functional link between hydraulic conductivity and scale. In any case one should note that the parameter estimations concerning the fractal models are not immediate, both in terms of knowledge of the fractal dimension itself and of other geometrical and/or physical parameters linked to it. This aspect is also related to the fact that most of the known fractal models were verified for homogeneous porous media. The use of empirical relationships, as power laws of sample-scaling type, can provide an early indication about the overall trends of the functional link between the hydraulic conductivity and porosity.

In this work, on the basis of data sets obtained by laboratory and field measurement methods, the scaling behaviour analysis of the hydraulic conductivity and effective porosity was performed for the relative characteristic measurement scales relative to a confined aquifer, characterized by a sandy loam porous media and considered homogeneous for the absence of stratification. This analysis, on the basis of a relation of a simple-scaling behavior, coupled with other fractal models such as suggested by $\mathrm{Xu}$ and $\mathrm{Yu}$ [36], Jacquin and Adler [12], and Muller and McCauley [41], shows that the scale index, for this specific soil type, is connected with the fractal dimension, which is determined directly considering the values obtained by the scaling law utilized. This analysis shows a new interpretation of the maximum pore size of the porous medium, starting from the considerations provided by $\mathrm{Xu}$ and $\mathrm{Yu}[36]$.

This direct approach, allowing the hydraulic conductivity to be related to the effective porosity, can be utilized to give a comparison parameter with the several fractal models used to describe the scaling behavior of the hydraulic conductivity and the effective porosity.

\section{The Role of Fractal Geometry in the Framework of Porosity and Hydraulic Conductivity}

The scaling behavior between the saturated hydraulic conductivity $(k)$ and the effective porosity $(\varphi)$ can be represented by the following power-law relationship [12]:

$$
k \approx \varphi^{\mu}
$$

where $\mu$ is the scale crowing index and $\varphi$ depends on the measurement scale $(s)$ and the pore size $(\lambda)$. This functional relation (1) is often associated with fractal models and therefore $\mu$, a function of the fractal dimension of pores $\left(D_{f}\right)$, can be proportional to the rate in which $D_{f}$ appears. For example, according to Jacquin and Adler [12], this result is proportional to the ratio $\left(4-D_{f}\right) /\left(2-D_{f}\right)$ for a model where the largest pores provide the pore-space connectivity. Other authors gave different function relations of the $\mu$ scale index, for example, Muller and McCauley [41], Korvin [42], and Giménez et al. [16]. In particular, in Muller and McCauley [41] this exponent is related to $\left(4-D_{f}\right) / D_{f}$ and is very close to that obtained by Jacquin and Adler [12].

From the theoretical point of view, the fractal dimension appears as one of the parameters that can be used in order to describe the porous medium. As such, it should occur in the expression of hydraulic conductivity, which is the only function of the geometry of the problem. In fact, according to Ahuja et al. [43] and Jacquin and Adler [12], the relation (1) is not retained sufficient in terms of cut-off limits and therefore the same may be generalized in the following functional relation:

$$
k=f\left(\varphi, D_{f}, \widehat{D}, \ldots\right)
$$

where $\widehat{D}$ is a spreading dimension, which involves a more complex scaling range. For these latter reasons the assessment of $\widehat{D}$, with each associated scaling behaviour, does not result immediately. In fact, as is well known in the literature [44], these scaling ranges are generally narrow, because these are 
determined by the type of fractal model used and by the same fractal analysis performed in two- and three-dimensional supports of the measurements (see also [41]). A fortiori, without predefining a fractal model, it is possible to perform a direct scaling analysis to obtain the same scale exponent, without explicitly stating the fractal dimension. This last approach, already considered in the literature $[10,11]$, is well suited to interpreting the experimental results, considering the possible presence of simple and multiscaling behaviours of the investigated parameter. Therefore, relation (1) can be expressed by the following empirical power law:

$$
k \approx a \varphi^{m}
$$

where $a$ is a coefficient depending on the specific porous media and $m$ is a general scale crowing index.

In terms of grain size distribution, the relation (3) is also clarified by the classical permeability-porosity relation of Kozeny [45], Carman [46], applied in various fields, such as groundwater flow, water/oil reservoirs, and so on. Recently $\mathrm{Xu}$ and $\mathrm{Yu}$ [36] developed a new form of the KozenyCarman relation for homogeneous porous media by fractal geometry, considering an expression of the porosity, in terms of the fractal dimension $\left(D_{f}\right)$ by exactly self-similar fractal geometry supports, namely, the Sierpinski carpet and gaskets, according to what is introduced by Muller and McCauley [41]. In this case the porosity relation, implicitly written in (1) and (3), is clarified by the following relation [47]:

$$
\varphi=\left(\frac{\lambda_{\min }}{\lambda_{\max }}\right)^{d_{E}-D_{f}},
$$

where $\lambda_{\min }$ and $\lambda_{\max }$, respectively, are equal to the minimum and maximum diameters of the pores characterizing the saturated porous medium, $d_{E}$ is the Euclidean dimension, which is equal to 2 and 3 in the two- and three-dimensional spaces, respectively. For the theoretical analysis, as suggested by $\mathrm{Xu}$ and $\mathrm{Yu}[36]$, according to (4), the pore area fractal dimension $D_{f}$ can be determined by

$$
D_{f}=d_{E}-\frac{\ln \varphi}{\ln \left(\lambda_{\min } / \lambda_{\max }\right)}
$$

while $\lambda_{\text {min }} \ll \lambda_{\text {max }}$ must be satisfied for fractal porous media. This latter aspect plays a fundamental role in the determination of the fractal dimension for the specific soil type and for the specific grain size distribution. The model of $\mathrm{Xu}$ and $\mathrm{Yu}$ [36] is essentially based on the characterization of the fractal dimension, $D_{f}$, and the tortuosity fractal dimension, $D_{T}$ $[23,35,37,48-50]$. In this model the relationship between the hydraulic conductivity and the effective porosity is expressed by the following relation:

$$
k=C_{f}\left(\frac{\varphi}{1-\varphi}\right)^{\left(1+D_{T}\right) / 2} \lambda_{\max }^{2}
$$

where the coefficient $C_{f}$ is equal to

$$
C_{f}=\frac{\left(\pi D_{f}\right)^{\left(1-D_{T}\right) / 2}\left[4\left(2-D_{f}\right)\right]^{\left(1+D_{T}\right) / 2}}{128\left(3+D_{T}-D_{f}\right)} .
$$

$\mathrm{Xu}$ and $\mathrm{Yu}[36]$ in the discussion of their model represented by (6), on the basis of a simple arrangement of solid particles for the maximum pore, give the following relation that allows determination of the maximum pore diameter, namely,

$$
\lambda_{\max }=d \sqrt{\frac{\varphi}{1-\varphi}}
$$

which is expressed as a function of particle diameter $d$ and porosity.

Relation (8), which is the basis of the $\mathrm{Xu}$ and $\mathrm{Yu}$ [36] model, assumes precise values from the experimental point of view, which can be investigated in the grain size distribution context of the porous medium considered. However, as will be shown later in this study, relation (8) is difficult to fit not considering the self-similarity space on the measurement scale of the effective porosity and not taking into account all the set of normalized values represented by the maximum pore distribution expressed in the same equation (8). The experimental evidence, in consideration of the hypothesis of self-similarity in the scale of $\varphi(s, \lambda)$, as will be shown for the case considered here, showed that the diameter $d$, taken as representative size of the particle, can be $d_{10}$, as well as being considered in many empirical and semiempirical formulas available in the literature $[45,46,51-55]$.

Regarding the tortuosity dimension, $D_{T}$, $\mathrm{Xu}$ and $\mathrm{Yu}[36]$ give the following relation for tortuous streamtubes in porous media:

$$
D_{T}=1+\frac{\ln \bar{\tau}}{\ln \left(L_{0} / \bar{\lambda}\right)},
$$

where the average tortuosity $\bar{\tau}$ is given by the results of Yu and Li [56] expressed by

$$
\bar{\tau}=\frac{1}{2}\left[1+\frac{1}{2} \sqrt{1-\varphi}+\sqrt{1-\varphi} \frac{\sqrt{(1 / \sqrt{1-\varphi}-1)^{2}+1 / 4}}{1-\sqrt{1-\varphi}}\right]
$$

and $L_{0}$ can be considered the upper cutoff or the upper limit of self-similarity, proportional to $\lambda_{\min }$, while $\bar{\lambda}$, namely, the average pore-capillary size, is given by [47]

$$
\bar{\lambda}=\frac{D_{f} \lambda_{\min }}{D_{f}-1} .
$$

Therefore, $\mathrm{Xu}$ and $\mathrm{Yu}$ [36] on the base of geometrical considerations give the following relation for the ratio $L_{0} / \bar{\lambda}$ :

$$
\frac{L_{0}}{\bar{\lambda}}=\frac{D_{f}-1}{D_{f}^{1 / 2}}\left[\frac{1-\varphi}{\varphi} \frac{\pi}{4\left(2-D_{f}\right)}\right]^{1 / 2} \frac{\lambda_{\max }}{\lambda_{\min }} .
$$

By this last relation it is possible to define the tortuosity dimension, $D_{T}$, expressed by (9) and then fall back on the hydraulic conductivity law defined by the relation (6).

The evaluation of the lower and upper cut-off limits within the relationships (4), (9), and (12) and in the same 
relation (6) is not of immediate determination, because there is an implicit dependence of the porosity on the values of $\lambda$ and in particular on the use of the relationship (8) as previously highlighted. This problem is more evident in the experimental measurements that show discrepancies already highlighted by Muller and McCauley [41] regarding the investigations conducted by Jacquin and Adler [12] and partially addressed by $\mathrm{Xu}$ and $\mathrm{Yu}$ [36] in the context of their theoretical and experimental investigations. In this framework the role of the measurement scale and the structure of the porous medium, and then the characteristic parameters influenced altogether by the medium heterogeneity locally or globally, are of fundamental importance for the choice of fractal models to use. In fact, this choice is also determined by potential simple and multiscaling behaviours of the same hydraulic conductivity, as well as the functional link $\varphi=$ $\varphi(s, \lambda)$ between the porosity, the measurement scale $(s)$, and the pore size $(\lambda)$.

Therefore, in this work it was deemed appropriate to proceed on the basis of simple-scaling considerations, evaluating the fractal behaviour of the hydraulic conductivity and effective porosity and comparing the models introduced by the above-cited authors and relation (3), which provides an immediate description of the experimental trend of the parameter considered for the aquifer under investigation and the values found by laboratory and field measurements, while retaining valid the self-similarity assumptions for the measurement scales observed.

\section{Experimental Data Setting}

In the present work the relationship (4) between hydraulic conductivity and effective porosity was experimentally verified $[10,13,14,17,20,57]$. For this purpose values of $k$ and $\varphi$ obtained by both field and laboratory measurements were taken into consideration, because the values of these parameters and their spatial variation do not depend on the specific method of measurement [8], but on the aquifer volume involved [58].

The field measurements were carried out on the confined aquifer of Montalto Uffugo (Italy) test field. This area has the geological characteristics of a recently formed valley, with conglomeratic and sandy alluvial deposits. Corresponding to the test field, after a sandy surface layer with a thickness of about $7 \mathrm{~m}$, one meets a clay lens with $4 \mathrm{~m}$ of thickness and then a layer of sand and silt depth up to $55 \mathrm{~m}$, where a bank of consolidated clay starts. The test field has eleven wells and two piezometers. The wells marked with odd numbers affect the aquifer under pressure, below the clay layer, and of these only well number 11 is completely penetrating, while the others reach $40 \mathrm{~m}$ in depth. The two piezometers $\mathrm{A}$ and $\mathrm{B}$ are both entirely penetrating in the confined aquifer. A stratigraphic and planimetrical layout of the test field area is shown in Figure 1.

A total of 67 values of $k$ and $\varphi$ were measured, 5 of these by tracer tests, 15 by slug tests, and other 15 by aquifer tests.

The tracer tests were performed all in forced flow conditions, using number 1 as the tracer inflow well and number
5 as the pumping and observation well. These two wells are $10 \mathrm{~m}$ apart. For all the tests $\mathrm{NaCl}$ was used as the tracer in well number 1 in a solution volume of $0.4 \mathrm{~m}^{3}$, with an $\mathrm{NaCl}$ concentration of $200 \mathrm{~kg} / \mathrm{m}^{3}$. The tracer inflow was performed in a short time for each test. The pumping rates were held constant during each tracer test considered, while the respective durations ranged between 5.4 and 34.84 days. The steady state conditions of the aquifer flow were verified for each tracer test and the drawdown-times data were analyzed by the Dupuis method (1863) to determine $k$. Moreover, the velocity of Darcy $\left(V_{D}\right)\left[\mathrm{LT}^{-1}\right]$, the correspondent effective velocity $(V)\left[\mathrm{LT}^{-1}\right]$, and the effective porosity $(\varphi)$ were determined [59].

All the slug tests were carried out following the guidelines suggested by Butler et al. [60] and Butler [61]. Therefore these were performed only on well number 11 and on piezometers A and B, all completely penetrating. The water volumes $V$ rapidly admitted in the columns during the tests ranged between $0.003 \mathrm{~m}^{3}$ and $0.040 \mathrm{~m}^{3}$ and the water level variations were measured by proper pressure transducers at fixed times [61]. Once the geometry of the system aquifer well is known, to determine $k$ and $\varphi$, the drawdown-time data sets obtained in this way were analyzed by the Cooper method [62].

For the aquifer tests, carried out in unsteady state conditions, the drawdown-time data were analyzed by the Neuman [63] and Jacob [64] methods, considering the initial and boundary conditions and the geometry of the system well known and taking into account that during the pumping the aquifer behaviour passed from confined to phreatic, because the aquifer proves to be under weak pressure. All the tests were performed to a constant pumping rate between 5.7 . $10^{-4} \mathrm{~m}^{3} / \mathrm{s}$ and $4.55 \cdot 10^{-3} \mathrm{~m}^{3} / \mathrm{s}$ and for time ranges between 23 and 94.8 hours. In this way it was possible to determine the hydraulic conductivity $(k)$, the storage coefficient $(S)$, and the effective porosity $(\varphi)$.

The laboratory measurements were carried out on 32 undisturbed soil samples, 18 of which were drawn out from the drilling column of piezometer A and number 14 from that of piezometer $B$, at several depths, between $11 \mathrm{~m}$ and $55 \mathrm{~m}$ from the ground surface. The hydraulic conductivity was measured for each of these samples, using flow cells as permeameter, and the effective porosity by the double weighting method. Further details about the measurement methodologies can be found in previous works [11].

\section{Results and Discussion}

The first step in the analysis of the hydraulic conductivity behavior as a function of porosity concerned the grouping of all the data sets obtained by the above-mentioned measurement methods.

The analysis of the experimental data highlights the scalar behavior of the hydraulic conductivity and effective porosity, albeit in a more evident manner for the first parameter and less marked for the second. However, for the analysis of the trend of these parameters with the scale, one can refer to previous studies relating to the same aquifer of the Montalto Uffugo test field [11]. 


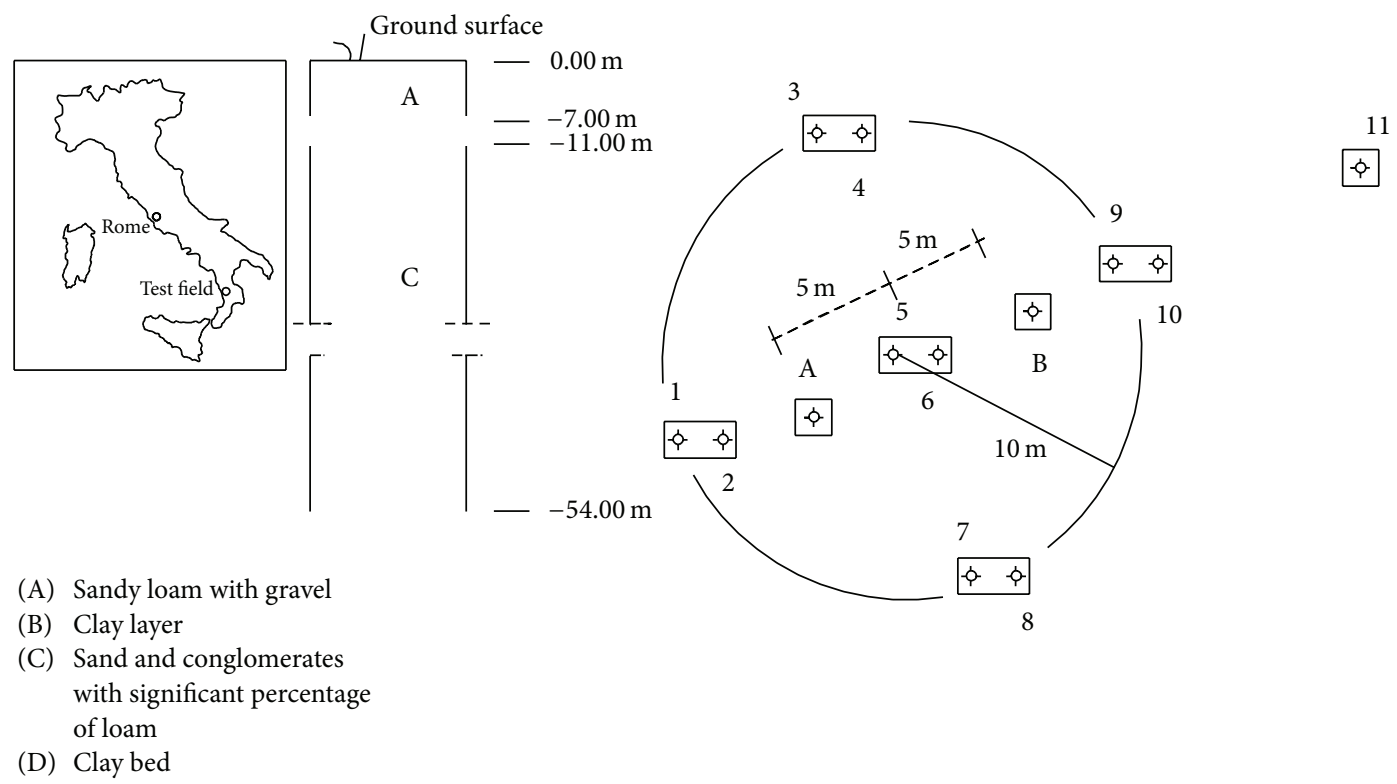

FIGURE 1: Stratigraphical and planimetrical layout of the test field.

Considering that both parameters $k$ and $\varphi$ are functions of the scale $s$, it is well known that there is a direct link between these two quantities; see, for example, the empirical relationships in the context of grain size distribution [45, 46], or in some fractal patterns [22]. Therefore, taking into account this analysis, at the laboratory scale pairs of values $(k, \varphi)$ were obtained, determined by the spatial variability of the sampling point in the thickness of the aquifer, along the vertical drillings of the piezometers considered. However, given that these measures are used in a context in which field measurements are also considered and because they provide values of $k$ and $\varphi$ averaged on the entire volume of the aquifer involved in the measurement, it was deemed appropriate, also for the measurements performed in the laboratory, to consider the corresponding mean values, resulting, respectively, $3.25 \cdot 10^{-7} \mathrm{~m} / \mathrm{s}$ for $k$ and $2.37 \cdot 10^{-2}$ for $\varphi$.

Similarly to what was performed for $k$ and $\varphi$ values measured in the laboratory, even for those obtained by slug tests, the mean values were considered for each data set relative, respectively, to piezometers $\mathrm{A}$ and $\mathrm{B}$ and to well number 11 (see Figure 1), showing different geometrical characteristics. For $k$ these mean that values were, respectively, found equal to $2.53 \cdot 10^{-6} \mathrm{~m} / \mathrm{s}$ for piezometers $\mathrm{A}$ and $\mathrm{B}$ and $2.66 \cdot 10^{-6} \mathrm{~m} / \mathrm{s}$ for well number 11, while for $\varphi$ the corresponding values were found equal to $5.44 \cdot 10^{-2}$ for piezometers $\mathrm{A}$ and $\mathrm{B}$ and $5.77 \cdot 10^{-2}$ for well number 11 .

The $k$ values obtained by tracer tests ranged between 1.83 . $10^{-6} \mathrm{~m} / \mathrm{s}$ and $6.00 \cdot 10^{-6} \mathrm{~m} / \mathrm{s}$, while the correspondent $\varphi$ values are in the range bounded by $4.50 \cdot 10^{-2}-8.26 \cdot 10^{-2}$.

Similarly, the $k$ values measured by aquifer tests ranged between $3: 28 \cdot 10^{-6} \mathrm{~m} / \mathrm{s}$ and $5.78 \cdot 10^{-6} \mathrm{~m} / \mathrm{s}$, while the correspondent $\varphi$ values are in the range bounded by 6.25 . $10^{-2}-9.77 \cdot 10^{-2}$.
Therefore, the scaling analysis relative to the values $k[\varphi(s)]$ was carried out, considering the relation (3) and estimating the scale parameters corresponding to this power law. In this case, relatively to all measurement scales taken into consideration, the value of the scale index $m$ was 1.753 , while the coefficient $a$ was 0.0004 , with a coefficient of determination of the interpolation law $R^{2}=0.887$, with a value of the root mean square error (RMSE) $8.903 \cdot 10^{-7}$. Figures 2(a) and 2(b) show the trends of the hydraulic conductivity evaluated as a function of the effective porosity, as well as the interpolation law of the power type corresponding to (4). Specifically Figure 2(b) show a scaling in which it is possible to observe the absence of cut-off limits and therefore to consider this behavior as a simple-scaling, allowing the selfsimilarity properties to be extended to the whole aquifer. For this reason the use of the models examined here is acceptable.

Furthermore, the trend of $k$ was also described by relation (1), taking as a coefficient $\mu$ the relations proposed by Jacquin and Adler [12] and Muller and McCauley [41].

In this case the nonlinear fitting procedure allowed the fractal dimension values to be determined for each of the proposed relationships. According to the first model, the value of the $\mu$ index was 5.066, with a value of the fractal dimension 1.508 and standard error 0.0051 . Considering the second model, the value of the $\mu$ index remained almost unchanged, while that of the fractal dimension was 0.659 , with a standard error value 0.0045 . For both models, the value of RMSE was $1.839 \cdot 10^{-6}$, namely, greater than that obtained using relationship (3). As an example, Figure 3 shows the fitting curve relative to the Muller and McCauley [41] model; this curve is almost coincident with that relative to the Jacquin and Adler [12] model. On the basis of the results obtained it can be said that the direct use of (3) allows, without the constraints resulting from the presence of the fractal dimension in the exponent $\mu$, a direct estimation 


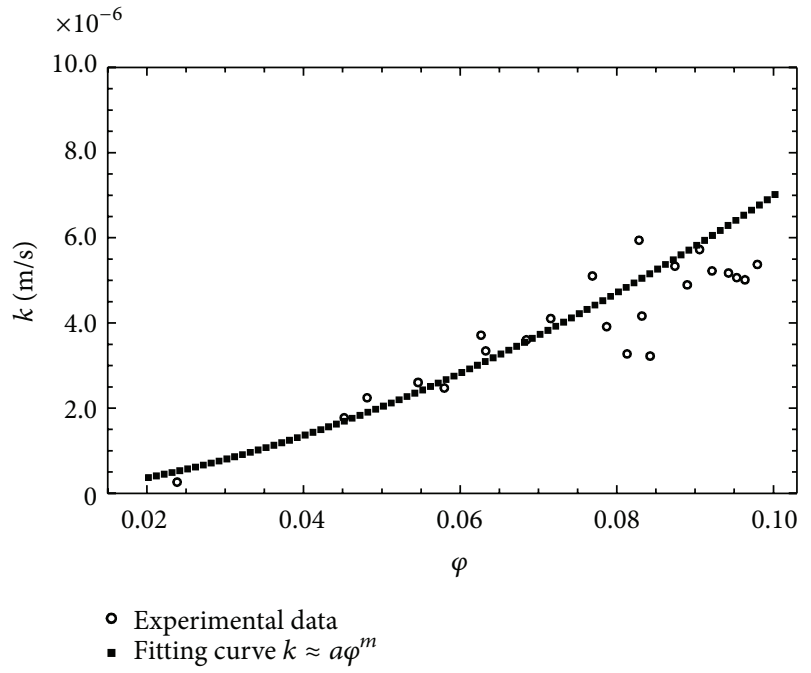

(a)

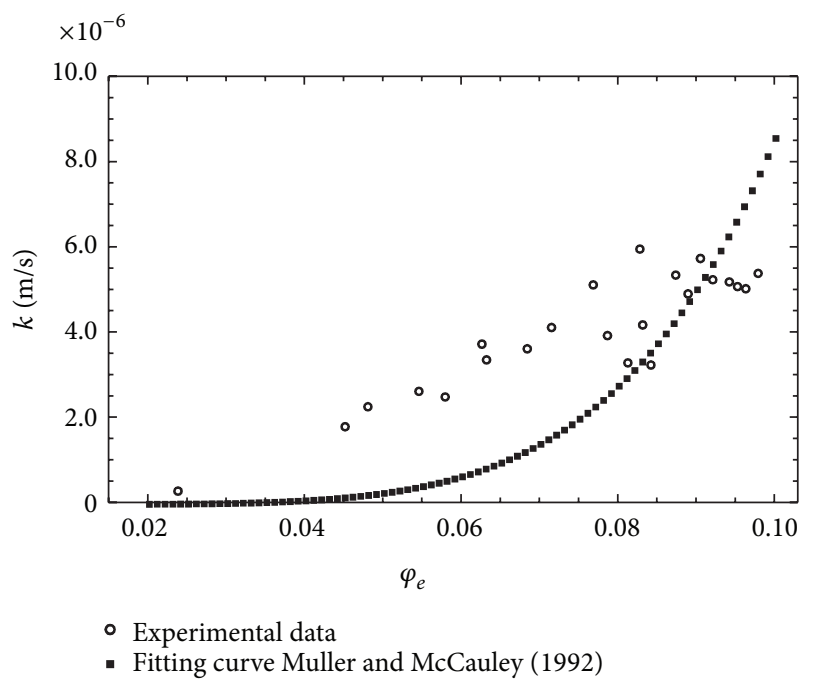

FIGURE 3: Fitting curve based on the Muller and McCauley [41] model.

of the general scale crowing index $m$ with a smaller value of RMSE. In order to investigate the link between the general scales crowing index and the fractal dimension, the model proposed by $\mathrm{Xu}$ and $\mathrm{Yu}$ [36] was considered, taking into account relation (6), according to which the hydraulic conductivity proves to be a function, besides of the effective porosity, of the fractal dimensions and tortuosity, expressed, respectively, by relations (5) and (9). One should consider that in the relationship proposed by $\mathrm{Yu}$ and $\mathrm{Li}$ [47] the link between the effective porosity and the fractal dimension is based exclusively on the determination of the minimum and maximum values of the pore diameters characterizing the saturated porous medium. Relation (5) provides, in fact, on the basis of relation (4), a parametric relationship in

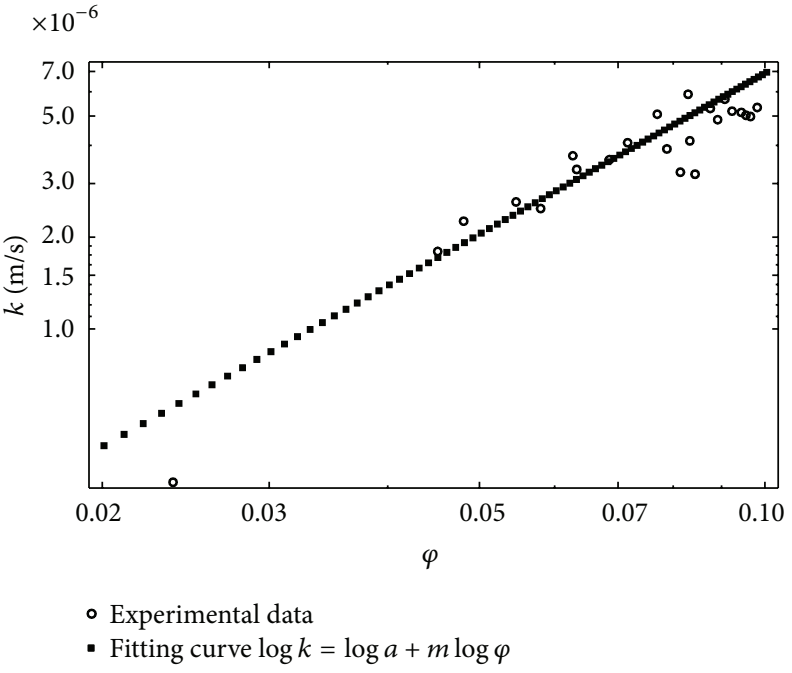

(b)

(4): (a) decimal scale; (b) logarithmical scale.

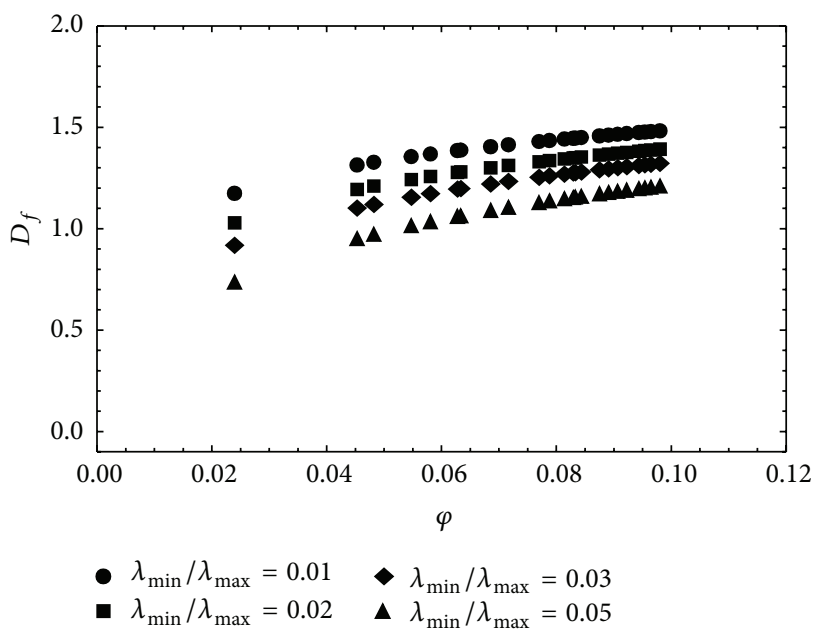

FIGURE 4: Relationship between fractal dimensions and porosity for different ratios $\lambda_{\min } / \lambda_{\max }$ according to $\mathrm{Xu}$ and $\mathrm{Yu}$ [36] model.

which $\lambda_{\min }$ and $\lambda_{\max }$ are in constant ratio according to the porous medium under consideration. In the present case four different values of the ratio $\lambda_{\min } / \lambda_{\max }$, that is, $0.01,0.02,0.03$, and 0.05 , were considered (see Figure 4 ). The peculiarity of the $\mathrm{Xu}$ and $\mathrm{Yu}$ [36] method allows in any case, on the basis of the grain size distribution, determination of the geometrical characteristics relating to the maximum pore diameter, as expressed by relation (8), and then determination, on the basis of geometric considerations [36], of the ratio $L_{0} / \bar{\lambda}$, all in consideration of relations (11) and (12), which are functions only of the fractal dimension. On other hand, regarding the estimation of the tortuosity dimension according to (9), this proves to be dependent, in addition to the aforementioned ratio $\lambda_{\min } / \lambda_{\max }$, even on the average tortuosity as expressed by relation (10). Therefore this step is of crucial importance, 


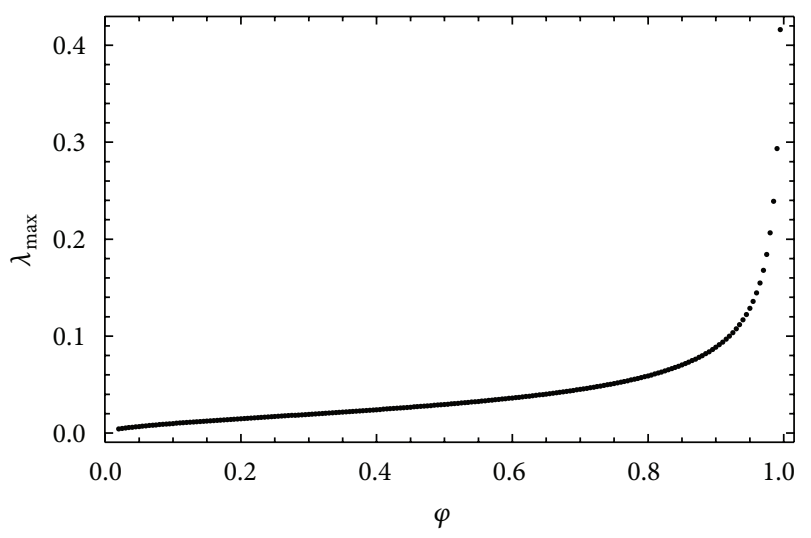

FIGURE 5: : Variation law of $\lambda_{\max }$ versus $\varphi$ for $d=0.041$

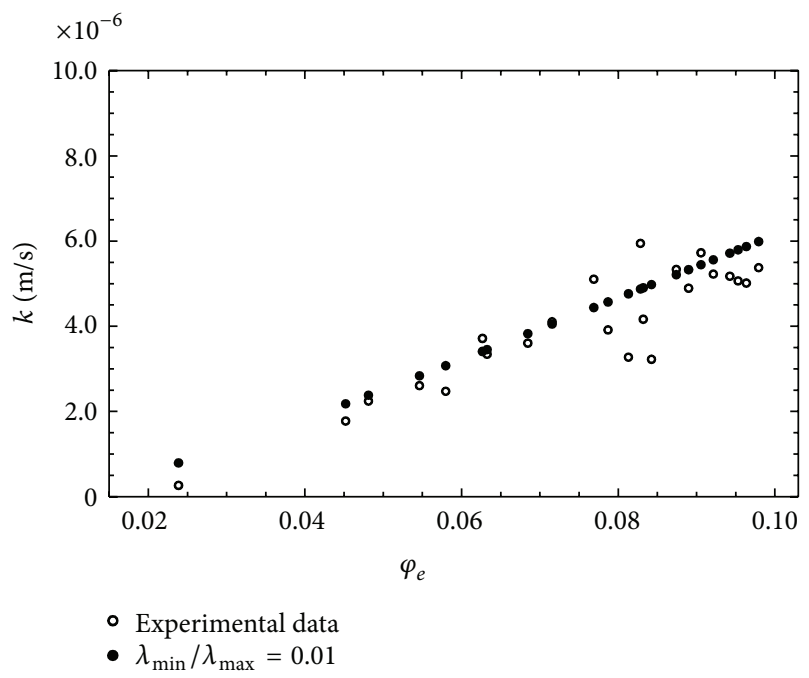

Figure 6: Fitting law according to the $\mathrm{Xu}$ and $\mathrm{Yu}$ [36] model for $\lambda_{\min } / \lambda_{\max }=0.01$.

on the basis of relationships (11) and (12), to assign the upper and lower limits of self-similarity in the scale of $\varphi(s, \lambda)$, then to characterize precisely the $\lambda_{\max }$ value expressed by (8) on which essentially the model of $\mathrm{Xu}$ and $\mathrm{Yu}$ [36] is based.

The experimental evidence in this case of sandy loam soil showed that for relationship (8) the value of $\lambda_{\max }$ must be researched in a range of porosity values exceeding those measured with the purpose of the convergence of the $\mathrm{Xu}$ and Yu [36] model. As regards the parameter $d$ a value was assumed of $0.041 \mathrm{~mm}$, obtained as the maximum of the $d_{10}$ values of the various soil samples extracted from the drilling columns of piezometers A and B and analyzed in the laboratory, assuming the particle size as effective grain diameter, of which $10 \%$ of the sample is finer. This assumption is justified because parameter $d$ affects proportionally the variation law of $\lambda_{\max }$, represented in Figure 5, which shows two different variation modes of this parameter with the porosity, that is, two different slopes of the representative curve. Furthermore, this curve represents the distribution law

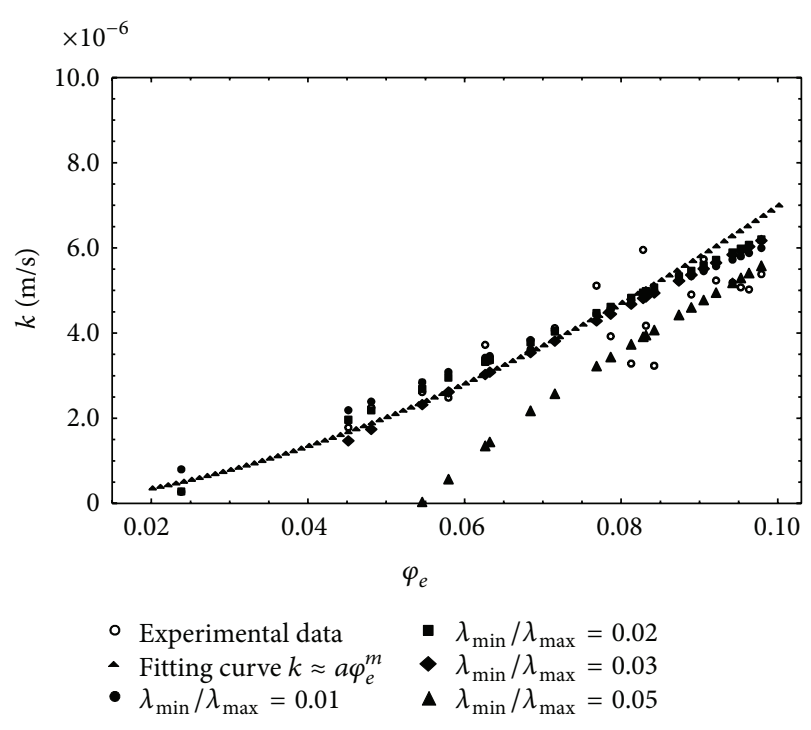

FIGURE 7: Fitting curves: for experimental and $\mathrm{Xu}$ and $\mathrm{Yu}$ [36] models.

of $\lambda_{\max }$ as a function of the porosity. Therefore, recalling (8), in consideration of the following integral:

$$
\int_{0}^{1} \lambda d \varphi=\int_{0}^{1} d \sqrt{\frac{\varphi}{1-\varphi}} d \varphi=\frac{d \pi}{2}
$$

it is possible to estimate the value of $d$ to be assumed in the $\mathrm{Xu}$ and $\mathrm{Yu}$ [36] model taking into account the proportionality constant $\pi / 2$.

Indeed, in this case the $d$ value, determined by relation (13), is equal to 0.041 , which is coincident with the maximum value of $d_{10}$, also given above. The value of the integral to the left in (13) represents the area under curve $\lambda_{\max }-\varphi$ and therefore allows approximate estimation of the value of $\lambda_{\max }$, corresponding to about $50 \%$ of this area, which in this case is 0.13 and this can be easily verified by the integral mean value theorem. This value allowed more accurate utilization of the $\mathrm{Xu}$ and $\mathrm{Yu}$ [36] model for a value of the rate $\lambda_{\min } / \lambda_{\max }$ of 0.01 . Regarding this ratio the fractal dimension value is next to $1.4-1.5$, as well as close to that estimated by the Jacquin and Adler [12] model. Figure 6 shows this interpolation law assessed on the basis of the experimental data. The RMSE value corresponding to this law is $6.984 \cdot 10^{-7}$, according to the data of Table 1, which is the lowest and closer to the experimental one obtained by (3). Figure 7 shows all the representative curves obtained by the $\mathrm{Xu}$ and $\mathrm{Yu}$ [36] model to vary the ratio $\lambda_{\min } / \lambda_{\max }$ in the range $0.01-0.05$, with the experimental values and the experimental fitting law represented by (3).

It should be pointed out that, on the basis of the experimental results, relation (3) gives in any case the opportunity to characterize the hydraulic conductivity behavior in a simple way and with sufficient reliability. This result allows the characteristic scale parameter to be obtained in a direct manner, without the use of a fractal model, which in any case requires the knowledge of a larger number of parameters. It is 
TABLE 1: Main parameter values of the fractal models analyzed.

\begin{tabular}{lccc}
\hline Models & $\mu$ & $D_{f}$ & RMSE \\
\hline Experimental power law & 1.753 & - & $8.903 \cdot 10^{-7}$ \\
Jacquin and Adler (1987) [12] & 5.066 & 1.508 & $1.839 \cdot 10^{-6}$ \\
Muller and McCauley (1992) [41] & 5.066 & 0.659 & $1.839 \cdot 10^{-6}$ \\
Xu and Yu (2008) ${ }^{*}[36]$ & - & $1.4-1.5$ & $6.984 \cdot 10^{-7}$ \\
\hline
\end{tabular}

${ }^{*}$ RMSE value is evaluated for $\lambda_{\min } / \lambda_{\max }=0.01$.

also noted that relation (3) gives an RMSE value less than that given by the models of Jacquin and Adler [12] and Muller and McCauley [41] and slightly higher than that of the $\mathrm{Xu}$ and $\mathrm{Yu}$ [36] model (see Table 1).

The methodology shown here, based essentially on the coupled use of relationship (3) and the $\mathrm{Xu}$ and $\mathrm{Yu}$ [36] model, may find a general use, extendable also to other soil types, while the values of the parameters $\mu$ and $m$ here obtained can be reasonably taken into consideration for soils belonging to the same class as that examined here.

\section{Conclusions}

The analysis of the direct scaling of the hydraulic conductivity and effective porosity was performed for a confined aquifer made up of a sandy-loam soil, considered homogeneous owing to the absence of stratifications. The measurements regarded the scale size of the parameters in relation to the laboratory, small, medium, and large field.

Based on the measurements carried out, the representativeness of the law of scaling was sought, according to both the scale and the functional link between the hydraulic conductivity and effective porosity, highlighting the simplescaling behavior, without considering however the high resolution field. This simplification, however, does not show in any case a trend representable by a multiscaling behavior.

The analysis of the scale index, obtained by appropriate laws of power type characterizing the aquifer taken into consideration, allows information about the fractal dimensions to be given indirectly in order to estimate using other specific models, considering only the physical scaling quantities, which, as is well known, are closely connected to the aforementioned power laws.

In this work a comparison between the experimental scaling law and some fractal models present in the literature was also considered. Specifically, the models of Jacquin and Adler [12], Muller and McCauley [41], and $\mathrm{Xu}$ and $\mathrm{Yu}$ [36] were considered. The results obtained showed a high degree of reliability of the experimental model represented by relation (3), compared to other models examined.

In fact, this power law model by direct scaling produced a value of the root mean square error smaller than that of the fractal models of Jacquin and Adler [12] and Muller and McCauley [41] and of the same order of magnitude as the fractal model of $\mathrm{Xu}$ and $\mathrm{Yu}$ [36]. About the use of this last model the behaviour of the variability of the largest diameter $\lambda_{\max }$ value was analysed as a function of the porosity measured. Specifically, greater details were provided about the value of this parameter to use for a given soil.

The model represented by relation (3) allows definition of the relationship between the variables under consideration in a simpler manner than that of the fractal models mentioned above, because it requires the consideration of a smaller number of parameters. In any case, using this approach in a coupled way with the model of $\mathrm{Xu}$ and $\mathrm{Yu}$ [36] can be advantageous, even in soils of a different type from the one considered here.

\section{References}

[1] S. P. Neuman, "Universal scaling of hydraulic conductivities and dispersivities in geologic media," Water Resources Research, vol. 26, no. 8, pp. 1749-1758, 1990.

[2] C. Clauser, "Permeability of crystalline rocks," Eos, Transactions American Geophysical Union, vol. 73, no. 21, pp. 233-238, 1992.

[3] H. Schad and G. Teutsch, "Effects of the investigation scale on pumping test results in heterogeneous porous aquifers," Journal of Hydrology, vol. 159, no. 1-4, pp. 61-77, 1994.

[4] S. P. Neuman, "Generalized scaling of permeabilities: validation and effect of support scale," Geophysical Research Letters, vol. 21, no. 5, pp. 349-352, 1994.

[5] C. W. Rovey II and D. S. Cherkauer, "Relation between hydraulic conductivity and texture in a carbonate aquifer: observations," Ground Water, vol. 32, no. 1, pp. 53-62, 1994.

[6] J. Guimerà, L. Vives, and J. Carrera, "A discussion of scale effects on hydraulic conductivity at a granitic site (El Berrocal, Spain)," Geophysical Research Letters, vol. 22, no. 11, pp. 1449-1452, 1995.

[7] D. Schulze-Makuch and D. S. Cherkauer, "Method developed for extrapolating scale behavior," Eos, Transactions American Geophysical Union, vol. 78, no. 13, 3, 7 pages, 1997.

[8] D. Schulze-Makuch and D. S. Cherkauer, "Variations in hydraulic conductivity with scale of measurement during aquifer tests in heterogeneous, porous carbonate rocks," Hydrogeology Journal, vol. 6, no. 2, pp. 204-215, 1998.

[9] D. Schulze-Makuch, D. A. Carlson, D. S. Cherkauer, and P. Malik, "Scale dependency of hydraulic conductivity in heterogeneous media," Ground Water, vol. 37, no. 6, pp. 904-919, 1999.

[10] C. Fallico, S. De Bartolo, S. Troisi, and M. Veltri, "Scaling analysis of hydraulic conductivity and porosity on a sandy medium of an unconfined aquifer reproduced in the laboratory," Geoderma, vol. 160, no. 1, pp. 3-12, 2010.

[11] C. Fallico, M. C. Vita, S. De Bartolo, and S. Straface, "Scaling effect of the hydraulic conductivity in a confined aquifer," Soil Science, vol. 177, no. 6, pp. 385-391, 2012.

[12] C. G. Jacquin and P. M. Adler, "Fractal porous media II: geometry of porous geological structures," Transport in Porous Media, vol. 2, no. 6, pp. 571-596, 1987.

[13] L. R. Ahuja, D. K. Cassel, R. R. Bruce, and B. B. Barnes, "Evaluation of spatial distribution of hydraulic conductivity using effective porosity data," Soil Science, vol. 148, no. 6, pp. 404-411, 1989.

[14] D. P. Franzmeier, "Estimation of hydraulic conductivity from effective porosity data for some Indiana soils," Soil Science Society of America Journal, vol. 55, no. 6, pp. 1801-1803, 1991.

[15] J. P. du Plessis and L. I. Roos, "Predicting the hydrodynamic permeability of sandstone with a pore-scale model," Journal of Geophysical Research, vol. 99, no. 10, pp. 19-776, 1994. 
[16] D. Giménez, R. R. Allmaras, D. R. Huggins, and E. A. Nater, "Prediction of the saturated hydraulic conductivity-porosity dependence using fractals," Soil Science Society of America Journal, vol. 61, no. 5, pp. 1285-1292, 1997.

[17] D. J. Timlin, L. R. Ahuja, Y. Pachepsky, R. D. Williams, D. Gimenez, and W. Rawls, "Use of Brooks-Corey parameters to improve estimates of saturated conductivity from effective porosity," Soil Science Society of America Journal, vol. 63, no. 5, pp. 1086-1092, 1999.

[18] H. Pape, C. Clauser, and J. Iffland, "Permeability prediction based on fractal pore-space geometry," Geophysics, vol. 64, no. 5, pp. 1447-1460, 1999.

[19] D. Giménez, W. J. Rawls, and J. G. Lauren, "Scaling properties of saturated hydraulic conductivity in soil," Geoderma, vol. 88, no. 3-4, pp. 205-220, 1999.

[20] L. E. Flint and J. S. Selker, "Use of porosity to estimate hydraulic properties of volcanic tuffs," Advances in Water Resources, vol. 26, no. 5, pp. 561-571, 2003.

[21] C. M. Regalado and R. Muñoz-Carpena, "Estimating the saturated hydraulic conductivity in a spatially variable soil with different permeameters: a stochastic Kozeny-Carman relation," Soil and Tillage Research, vol. 77, no. 2, pp. 189-202, 2004.

[22] A. Costa, "Permeability-porosity relationship: a reexamination of the Kozeny-Carman equation based on a fractal pore-space geometry assumption," Geophysical Research Letters, vol. 33, no. 2, Article ID L02318, 2006.

[23] S. W. Wheatcraft and S. W. Tyler, "An explanation of scaledependent dispersivity in heterogeneous aquifers using concepts of fractal geometry," Water Resources Research, vol. 24, no. 4, pp. 566-578, 1988.

[24] J. Carrera, "An overview of uncertainties in modelling groundwater solute transport," Journal of Contaminant Hydrology, vol. 13, no. 1-4, pp. 23-48, 1993.

[25] D. Mallants, B. P. Mohanty, A. Vervoort, and J. Feyen, "Spatial analysis of saturated hydraulic conductivity in a soil with macropores," Soil Technology, vol. 10, no. 2, pp. 115-131, 1997.

[26] N. R. A. Bird and E. Perrier, "Multiscale percolation properties of a fractal pore network," Geoderma, vol. 160, no. 1, pp. 105-110, 2010.

[27] C. Knudby and J. Carrera, "On the use of apparent hydraulic diffusivity as an indicator of connectivity," Journal of Hydrology, vol. 329, no. 3-4, pp. 377-389, 2006.

[28] A. J. Katz and A. H. Thompson, "Fractal sandstone pores: implications for conductivity and pore formation," Physical Review Letters, vol. 54, no. 12, pp. 1325-1328, 1985.

[29] C. E. Krohn and A. H. Thompson, "Fractal sandstone pores: automated measurements using scanning-electron-microscope images," Physical Review B, vol. 33, no. 9, pp. 6366-6374, 1986.

[30] G. Daccord and R. Lenormand, "Fractal patterns from chemical dissolution," Nature, vol. 325, no. 6099, pp. 41-43, 1987.

[31] A. H. Thompson, A. J. Katz, and C. E. Krohn, "Microgeometry and transport properties of sedimentary rock," Advances in Physics, vol. 36, no. 5, pp. 625-694, 1987.

[32] C. E. Krohn, "Fractal measurements of sandstones, shales, and carbonates," Journal of Geophysical Research, vol. 93, no. 4, pp. 3297-3305, 1988.

[33] P. M. Doyen, "Permeability, conductivity, and pore geometry of sandstone," Journal of Geophysical Research, vol. 93, no. 7, pp. 7729-7740, 1988.

[34] Y. Bernabé and C. Bruderer, "Effect of the variance of pore size distribution on the transport properties of heterogeneous networks," Journal of Geophysical Research B, vol. 103, no. 1, pp. 513-525, 1998.

[35] B. Yu and P. Cheng, "A fractal permeability model for bidispersed porous media," International Journal of Heat and Mass Transfer, vol. 45, no. 14, pp. 2983-2993, 2002.

[36] P. Xu and B. Yu, "Developing a new form of permeability and Kozeny-Carman constant for homogeneous porous media by means of fractal geometry," Advances in Water Resources, vol. 31, no. 1, pp. 74-81, 2008.

[37] R. P. Ewing, Q. Hu, and C. Liu, "Scale dependence of intragranular porosity, tortuosity, and diffusivity," Water Resources Research, vol. 46, no. 6, Article ID W06513, 12 pages, 2010.

[38] D. Schulze-Makuch, Facies dependent scale behavior of hydraulic conductivity and longitudinal dispersivity in the carbonate aquifer of southeastern Wisconsin [Ph.D. thesis], University of Wisconsin-Milwaukee, 1996.

[39] C. W. Rovey II, "Assessing flow systems in carbonate aquifers using scale effects in hydraulic conductivity," Environmental Geology, vol. 24, no. 4, pp. 244-253, 1994.

[40] P. Meakin, Fractals, Scaling and Growth Far From Equilibrium, Cambridge University Press, Cambridge, UK, 1998.

[41] J. Muller and J. L. McCauley, "Implication of fractal geometry for fluid flow properties of sedimentary rocks," Transport in Porous Media, vol. 8, no. 2, pp. 133-147, 1992.

[42] G. Korvin, Fractal Models in the Earth Sciences, Elsevier Science, Oxford, UK, 1992.

[43] L. R. Ahuja, J. W. Naney, R. E. Green, and D. R. Nielsen, "Macroporosity to characterize spatial variability of hydraulic conductivity and effects of land management," Soil Science Society of America Journal, vol. 48, no. 4, pp. 699-702, 1984.

[44] O. Malcai, D. A. Lidar, O. Biham, and D. Avnir, "Scaling range and cutoffs in empirical fractals," Physical Review E, vol. 56, no. 3, pp. 2817-2828, 1997.

[45] J. Kozeny, "Über kapillare Leitung des Wassers in Boden," Sitzungsberichte der Wiener Akademie des Wissenschaften, vol. 2, pp. 136-306, 1927 (German).

[46] P. C. Carman, "Fluid flow through granular beds", Transactions of the Institution of Chemical Engineers, vol. 15, pp. 150-166, 1937.

[47] B. Yu and J. Li, "Some fractal characters of porous media," Fractals, vol. 9, no. 3, pp. 365-372, 2001.

[48] N. Epstein, "On tortuosity and the tortuosity factor in flow and diffusion through porous media," Chemical Engineering Science, vol. 44, no. 3, pp. 777-779, 1989.

[49] A. Majumdar and B. Bhushan, "Role of fractal geometry in roughness characterization and contact mechanics of surfaces," Journal of Tribology, vol. 112, no. 2, pp. 205-216, 1990.

[50] B.-M. Yu, "Fractal character for tortuous streamtubes in porous media," Chinese Physics Letters, vol. 22, no. 1, pp. 158-160, 2005.

[51] C. S. Slichter, "Theoretical investigations of the motion of ground waters," U.S. Geological Survey 19th Annual Report, Part 2, 1899.

[52] A. M. Amer and A. A. Awad, "Permeability of cohesionless soils," Journal of Geotechnical and Geoenvironmental Engineering, vol. 100, no. 12, pp. 1309-1316, 1974.

[53] M. S. Alyamani and Z. Sen, "Determination of hydraulic conductivity from complete grain-size distribution curves," Ground Water, vol. 31, no. 4, pp. 551-555, 1993.

[54] J. Odong, "Evaluation of empirical formulae for determination of hydraulic conductivity based on grain-size analysis," Journal of American Science, vol. 3, pp. 54-60, 2007. 
[55] C. Cheng and X. Chen, "Evaluation of methods for determination of hydraulic properties in an aquifer-aquitard system hydrologically connected to a river," Hydrogeology Journal, vol. 15, no. 4, pp. 669-678, 2007.

[56] B.-M. Yu and J.-H. Li, "A geometry model for tortuosity of flow path in porous media," Chinese Physics Letters, vol. 21, no. 8, pp. 1569-1571, 2004.

[57] T. Vienken and P. Dietrich, "Field evaluation of methods for determining hydraulic conductivity from grain size data," Journal of Hydrology, vol. 400, no. 1-2, pp. 58-71, 2011.

[58] X. Sánchez-Vila, J. Carrera, and J. P. Girardi, "Scale effects in transmissivity," Journal of Hydrology, vol. 183, no. 1-2, pp. 1-22, 1996.

[59] J. Bear, Hydraulics of Groundwater, McGraw-Hill, New York, NY, USA, 1972.

[60] J. J. Butler Jr., C. D. McElwee, and W. Liu, "Improving the quality of parameter estimates obtained from slug tests," Ground Water, vol. 34, no. 3, pp. 480-490, 1996.

[61] J. J. Butler Jr, The Design, Performance, and Analysis of Slug Tests. Kansas Geological Survey, The University of Kansas, Lewis Publishers, 1997.

[62] H. H. Cooper, J. D. Bredehoeft, and I. S. Papadopulos, "Response of a finite-diameter well to an instantaneous charge of water," Water Resources Research, vol. 3, p. 263, 1967.

[63] S. P. Neuman, "Theory of flow in unconfined aquifers considering delayed response of the water table," Water Resources Research, vol. 8, pp. 1031-1045, 1972.

[64] C. E. Jacob, "On the flow of water in an elastic artesian aquifer," Transactions, American Geophysical Union, vol. 21, no. 2, pp. 574-586, 1940. 

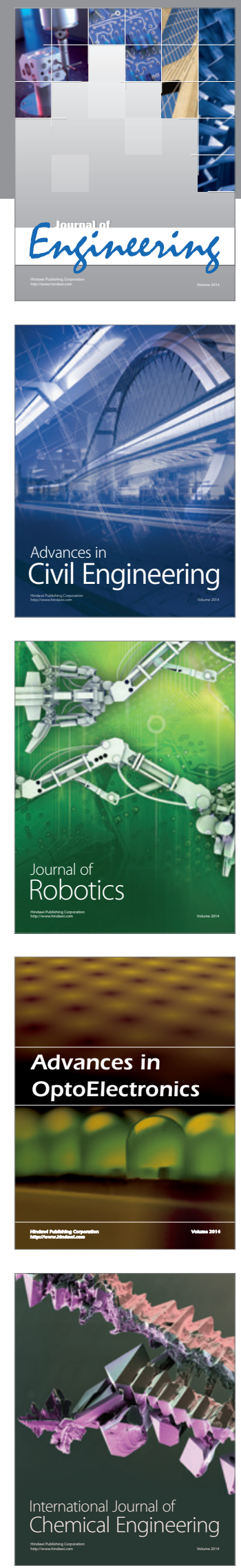

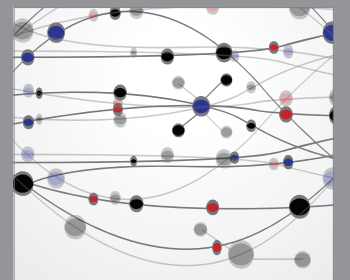

The Scientific World Journal
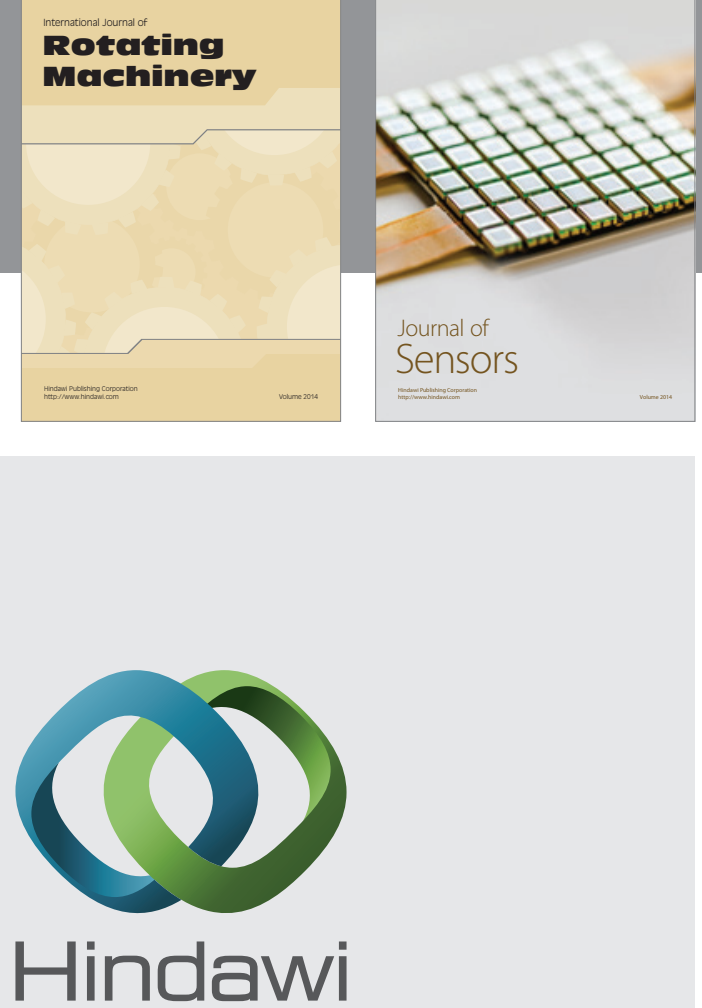

Submit your manuscripts at http://www.hindawi.com
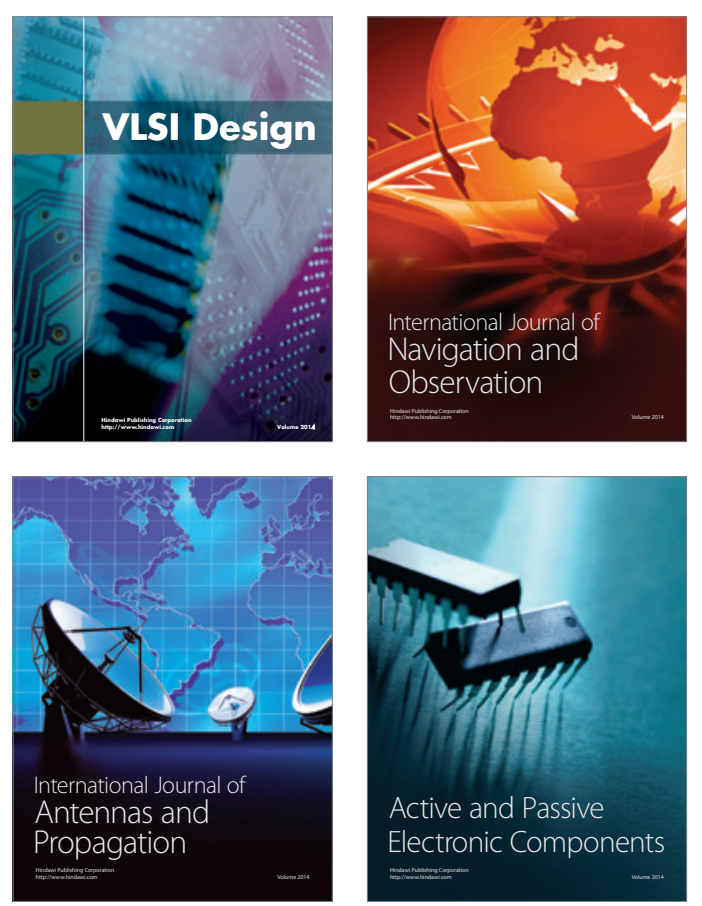
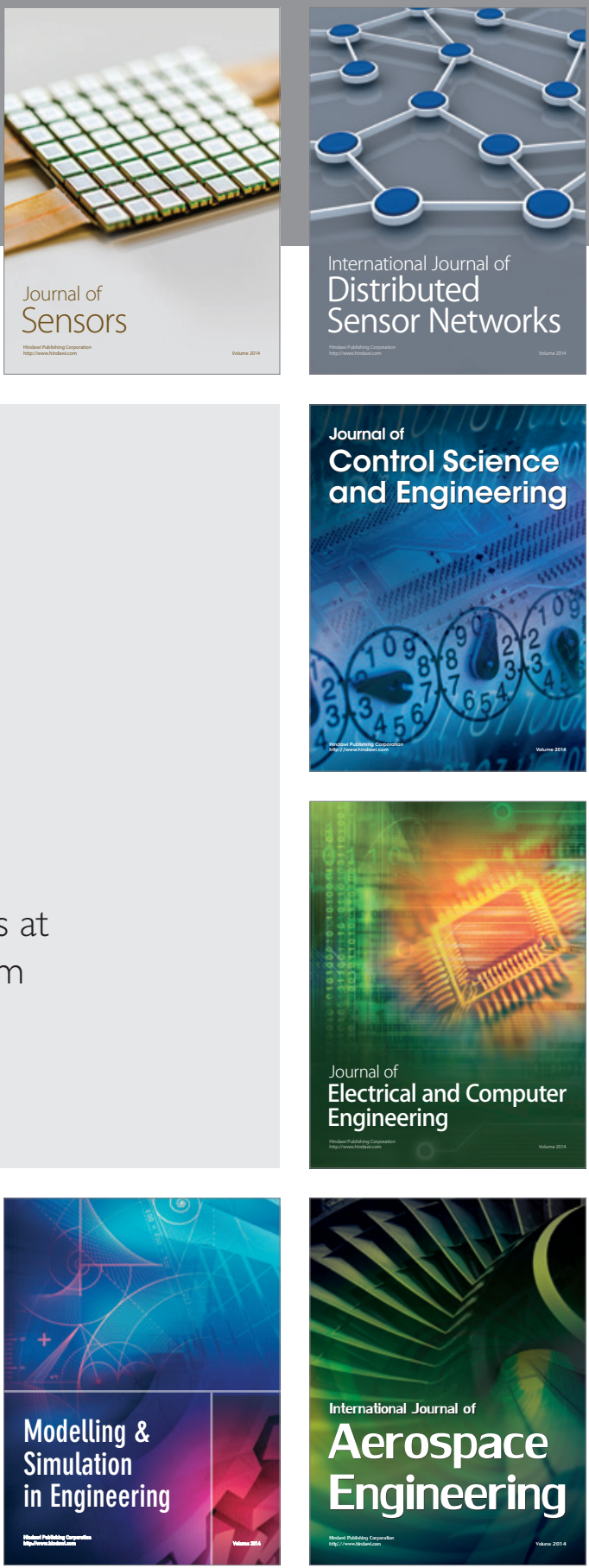

Journal of

Control Science

and Engineering
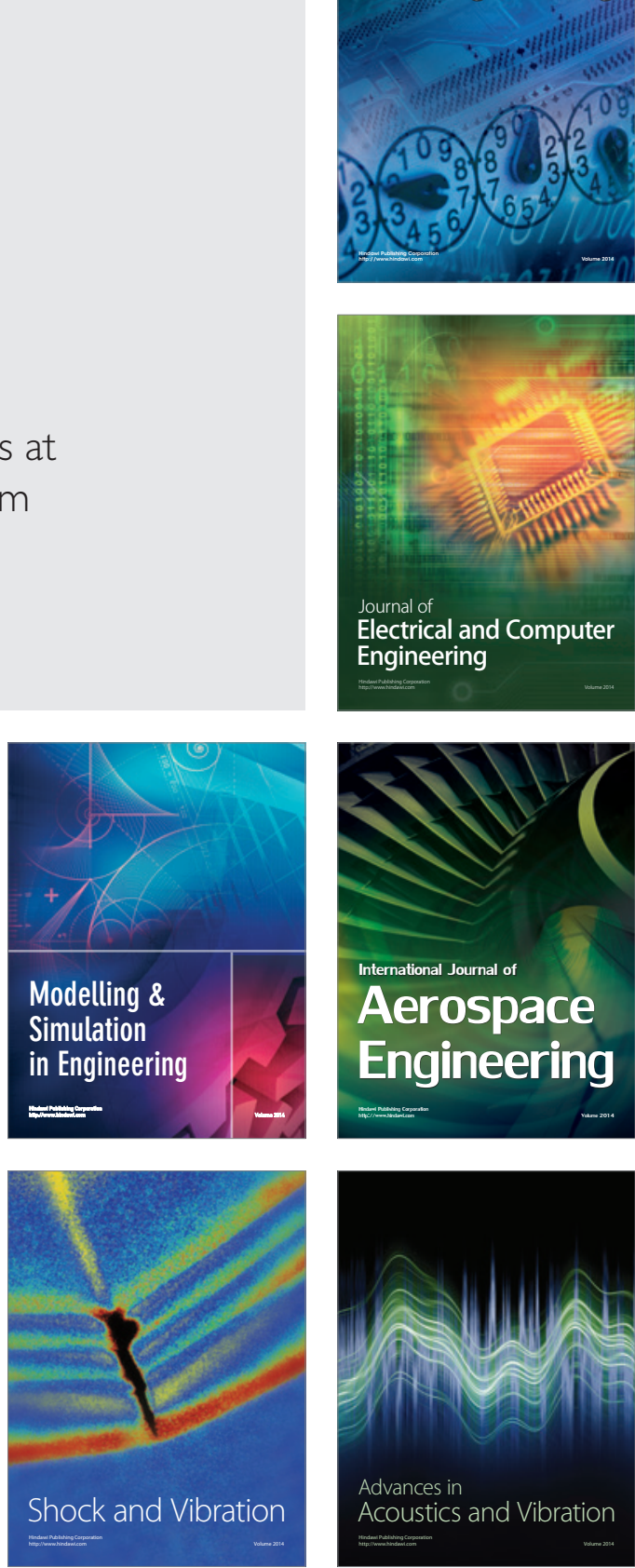